\title{
Far-infrared amide IV-VI spectroscopy of isolated 2- and 4-Methylacetanilide
}

Cite as: J. Chem. Phys. 145, 104309 (2016); https://doi.org/10.1063/1.4962360

Submitted: 12 June 2016 . Accepted: 26 August 2016 . Published Online: 13 September 2016

Vasyl Yatsyna (D), Daniël J. Bakker, Raimund Feifel, Anouk M. Rijs, and Vitali Zhaunerchyk

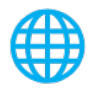

\section{ARTICLES YOU MAY BE INTERESTED IN}

A consistent and accurate ab initio parametrization of density functional dispersion correction (DFT-D) for the 94 elements $\mathrm{H}-\mathrm{Pu}$

The Journal of Chemical Physics 132, 154104 (2010); https://doi.org/10.1063/1.3382344

Infrared/ultraviolet quadruple resonance spectroscopy to investigate structures of electronically excited states

The Journal of Chemical Physics 136, 114202 (2012); https://doi.org/10.1063/1.3693508

The electronic spectrum of the amino acid tryptophan in the gas phase

The Journal of Chemical Physics 84, 2534 (1986); https://doi.org/10.1063/1.450323

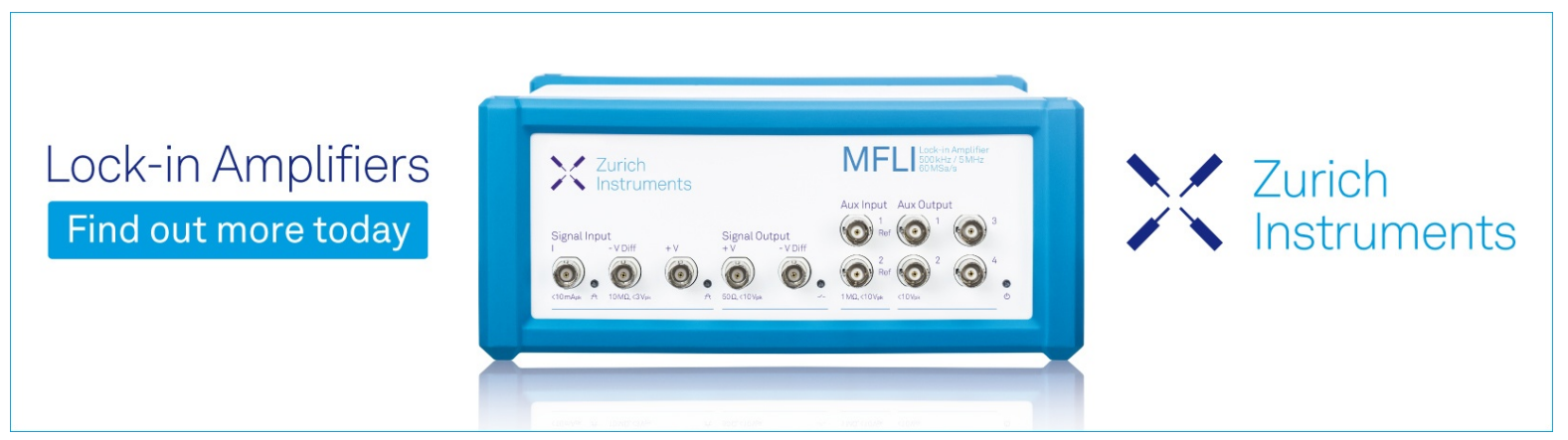




\title{
Far-infrared amide IV-VI spectroscopy of isolated 2- and 4-Methylacetanilide
}

\author{
Vasyl Yatsyna, ${ }^{1,2}$ Daniël J. Bakker, ${ }^{2}$ Raimund Feifel, ${ }^{1}$ Anouk M. Rijs, ${ }^{2, a)}$ \\ and Vitali Zhaunerchyk ${ }^{1, b)}$ \\ ${ }^{1}$ Department of Physics, University of Gothenburg, 41296 Gothenburg, Sweden \\ ${ }^{2}$ Institute for Molecules and Materials, FELIX Laboratory, Radboud University, Toernoovield 7-c, \\ 6525 ED Nijmegen, The Netherlands
}

(Received 12 June 2016; accepted 26 August 2016; published online 13 September 2016)

\begin{abstract}
Delocalized molecular vibrations in the far-infrared and $\mathrm{THz}$ ranges are highly sensitive to the molecular structure, as well as to intra- and inter-molecular interactions. Thus, spectroscopic studies of biomolecular structures can greatly benefit from an extension of the conventional mid-infrared to the far-infrared wavelength range. In this work, the conformer-specific gas-phase far-infrared spectra of two aromatic molecules containing the peptide-CO-NH- link, namely, 2- and 4-Methylacetanilide, are investigated. The planar conformations with trans configuration of the peptide link have only been observed in the supersonic-jet expansion. The corresponding far-infrared signatures associated with the vibrations of the peptide - CO-NH- moiety, the so-called amide IV-VI bands, have been assigned and compared with the results of density functional theory frequency calculations based on the anharmonic vibrational second-order perturbation theory approach. The analysis of the experimental and theoretical data shows that the amide IV-VI bands are highly diagnostic for the geometry of the peptide moiety and the molecular backbone. They are also strongly blue-shifted upon formation of the $\mathrm{NH} \cdots \mathrm{O}-\mathrm{C}$ hydrogen bonding, which is, for example, responsible for the formation of secondary protein structures. Furthermore, the amide IV-VI bands are also diagnostic for the cis configuration of the peptide link, which can be present in cyclic peptides. The experimental gas-phase data presented in this work can assist the vibrational assignment of similar biologically important systems, either isolated or in natural environments. Published by AIP Publishing. [http://dx.doi.org/10.1063/1.4962360]
\end{abstract}

\section{INTRODUCTION}

The knowledge about the three-dimensional (3D) structure of biological molecules is highly important, as the structure typically controls biological processes and chemical reactions, molecular recognition and selective binding of ligands, molecular transport properties, etc. Hence, the development of new or complementary approaches for molecular structural analysis is highly relevant for broadening and deepening our understanding of biological functioning. Infrared (IR) spectroscopy that probes molecular vibrations is a suitable tool to study the molecular structure. In the last decades, mid-IR studies of biomolecular building blocks in combination with quantum chemical calculations were successfully implemented for structural analysis of various biologically important molecules. ${ }^{1}$ For polypeptides and proteins, typically the amide $\mathrm{A}$ ( $\mathrm{NH}$ stretch) and amide I-III (C-O stretch and $\mathrm{NH}$ bend) mid-IR vibrations are studied, since these vibrations are characterized by strong absorption intensity and are sensitive to the local electrostatic environment of peptide links, as well as the presence of hydrogen bonding, etc. In contrast, the far-IR vibrations have delocalized character, which makes them suited for the analysis of global 3D structure of biomolecules. Moreover, the vibrational motions corresponding to weak interactions

\footnotetext{
a)Electronic mail: a.rijs@science.ru.nl

b) Electronic mail: vitali.zhaunerchyk@physics.gu.se
}

such as hydrogen bonding and van der Waals interactions are located in the low-frequency far-IR region, enabling their direct studies by means of far-IR spectroscopy. ${ }^{2}$ With the development of more efficient radiation sources, spectroscopy in the far-IR (or THz) spectral range becomes more accessible and promising for applications in different research areas, such as studies of the structure and dynamics of peptides and proteins, and the hydration layer around biomolecules. ${ }^{3-6}$

Among the early far-IR studies of biologically important molecules, it is worth to mention $N$-Methylacetamide (NMA), which contains a peptide link and two alpha carbons, making it the simplest model for a peptide backbone. Based on far-IR spectra and normal mode analysis of NMA, ${ }^{7}$ more complex model systems were studied. In particular, the fundamental amide vibrations in the far-IR range, the so-called amide IV-VII bands (see Fig. 1(b)), were recognized and shown to be characteristic for peptides and proteins. ${ }^{8-14}$ These bands are observed in the range of $200-800 \mathrm{~cm}^{-1}$, with amide $\mathrm{V}$ being the strongest one, corresponding to the NH out-of-plane (o.p.) bending motion. Although several early condensed-phase studies had demonstrated that the amide $\mathrm{V}$ bands have a strong sensitivity to the secondary structures of polypeptides, ${ }^{9,15}$ the subsequent IR studies were mostly focused on the amide $\mathrm{A}$ and amide I-III bands, mainly because they are amenable to highly accurate theoretical treatment, and are more straightforward to study experimentally. Only few recent far-IR studies were devoted to amide IV-VII range in the condensed-phase , $^{4-16}$ and in the gas-phase. ${ }^{19-23}$ With the advance of powerful far-IR 
4-MA
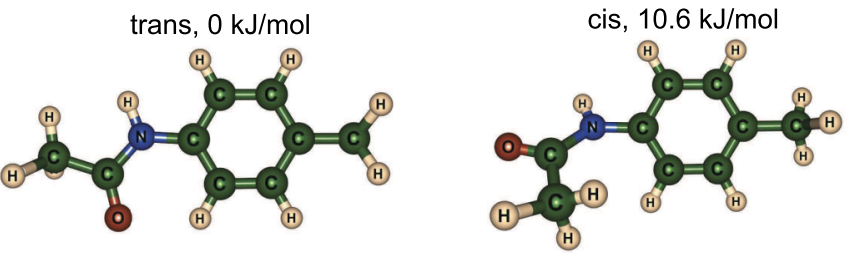

(a)
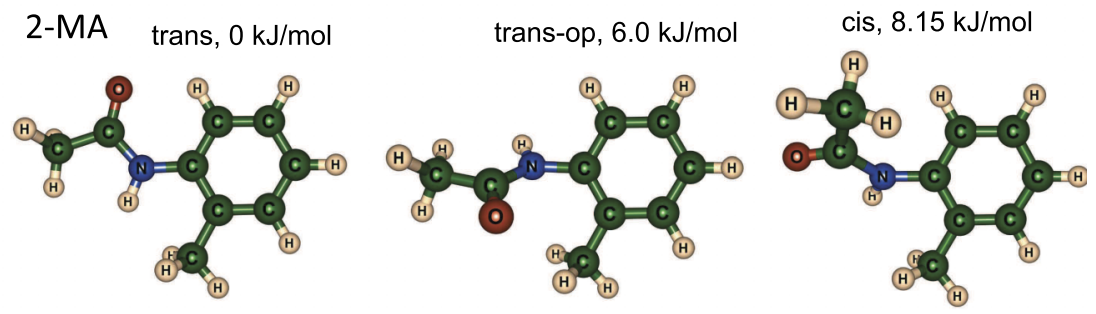
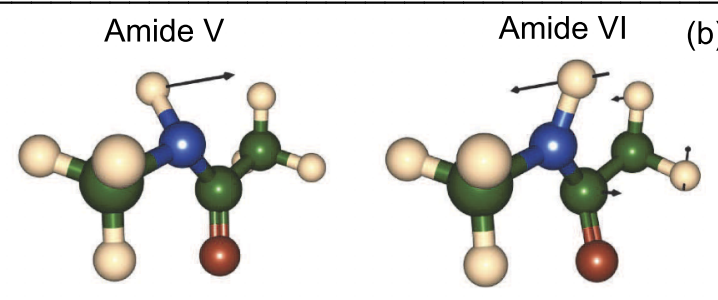

FIG. 1. (a) Geometries and corresponding energies of the 4-MA and 2-MA stable conformations, calculated with the B3LYP functional and N07D basis set. The energy of the conformations is shown with respect to the trans ones. (b) Graphical representation of the amide IV-VI normal modes of NMA, shown with scaled vectors of vibrational displacement. Amide IV vibrations involve $\mathrm{CO}$ in-plane (i.p.) bending, $\mathrm{CC}$ stretch, and $\mathrm{CNC}$ deformation; amide $\mathrm{V}$ is mostly $\mathrm{NH}$ out-ofplane bending; amide VI is mostly $\mathrm{CO}$ out-of-plane bending with some out-ofplane displacement of the $\mathrm{NH}$ group.
$(\mathrm{THz})$ radiation sources and the development of efficient theoretical approaches which enable accurate calculations of low-frequency vibrational motions, ${ }^{22-24}$ far-IR spectroscopy can nowadays serve as a highly informative extension to the well-established mid-IR region for the studies of molecular structure and weak interactions. For example, in the case of Phe-Ala and Phe-Gly dipeptides, the far-IR spectra enabled the distinction between two conformations which were not possible to be resolved in the mid-IR. ${ }^{22}$ In the case of Phe-Pro dipeptides, ${ }^{23}$ the combination of far-IR spectra with $a b$ initio molecular dynamics simulations enabled the study of the $\beta$ - and $\gamma$-turn conformations. The most conformerspecific bands in that case were associated with NH o.p. bending motions, either as a part of the $\mathrm{NH}_{2}$ moiety or the peptide - $\mathrm{CO}-\mathrm{NH}-$ moiety.

In the current work, we study low-frequency peptide moiety vibrations in isolated 2- and 4-Methylacetanilide (2-MA and 4-MA) molecules. We employ the free-electron laser FELIX, delivering tunable coherent radiation in the far-IR, and the IR-UV ion-dip spectroscopy technique to measure the conformer-specific 2-MA and 4-MA spectra in the gas phase. The experimental measurements have been combined with theoretical calculations to assign the lowfrequency vibrations of peptide moiety and to investigate their structure-specificity. The 2-MA and 4-MA molecules are substituted acetanilides ( $N$-phenylamides), in which the peptide-CO-NH- moiety links a methyl group with a substituted aromatic ring (methylphenyl group). Two different isomeric configurations were studied, 2-MA and 4-MA (see Fig. 1(a)). In general, the - $\mathrm{CO}-\mathrm{NH}-$ moiety can adopt two distinct nearly planar configurations, either trans or cis. In the case of 4-MA, this results in two geometries (see Fig. 1(a)), which have a significant difference in energy. In the case of 2-MA, where the substituent methyl group is located next to the acetamide group, steric interactions lead to the existence of the third conformer, with the - $\mathrm{CO}-\mathrm{NH}-$ moiety of trans character, but oriented out of plane with respect to the aromatic ring. This conformation is characterized by a nonzero dihedral angle $\left(\approx 60^{\circ}\right)$ between the $-\mathrm{CO}-\mathrm{NH}-$ moiety and the ring planes, and we call it trans-op 2-MA in what follows (Fig. 1(a)). Moreover, in the trans-op conformation of 2-MA, the -CO-NH- moiety deviates from planarity by $\approx 10^{\circ}$ (B3LYP/N07D geometry). Since in proteins deviation from planarity is quite common, ${ }^{25,26}$ the influence of this structural feature on the far-IR spectrum is of great interest.

\section{EXPERIMENTAL TECHNIQUE}

The mid-IR and far-IR spectra of 4-MA and 2-MA molecules were measured utilizing IR-UV ion-dip spectroscopy. ${ }^{27}$ Briefly, in this technique, the molecules are prepared in the electronic- and vibrational-ground states by means of supersonic-jet cooling and are excited by IR photons and then probed with UV photons. The UV photon energy is selected to ionize the molecules in form of resonant-enhanced multiphoton ionization (REMPI). Upon the resonant IR excitation the vibronic ground state is depleted, which results in a reduction of the ion signal produced by REMPI. The conformer-specific IR spectra can be measured by scanning the frequency of the IR laser while the frequency of the UV laser is set to the specific REMPI transition of the selected molecular conformation. The 4-MA and 2-MA samples used for the present study were obtained commercially with a stated purity of $99 \%$ (Sigma-Aldrich) and $>98 \%$ (Apollo Scientific), respectively. A pulsed molecular beam source with a heated sample compartment and heated pulsed valve (Parker general valve Series 9) was used to deliver the molecules 
into the interaction region. The 4-MA and 2-MA samples were heated to $95^{\circ} \mathrm{C}$ and $75^{\circ} \mathrm{C}$, respectively, while the temperature of the pulsed valve was kept $5-10^{\circ}$ higher in order to avoid condensation of the sample in the valve. Argon at high pressure, 3 bars, was used as a carrier gas. After the supersonic jet expansion, the coldest part of the molecular beam was selected with a skimmer. Tunable UV radiation was produced by a frequency doubled dye laser (NarrowScan, Radiant Dyes) pumped by a frequency tripled YAG laser (Spectra Physics). The molecular parent ions, generated in the interaction region by a $(1+1)$-REMPI scheme, were detected with a Jordan reflectron time-of-flight mass spectrometer. The IR light pulses preceding the UV radiation were generated by the free electron laser FELIX operating at $10 \mathrm{~Hz}$. The repetition rate of the UV laser was twice higher $(20 \mathrm{~Hz})$, providing reference measurements without IR radiation at every second UV pulse. The IR spectra were measured in the range of $220-1800 \mathrm{~cm}^{-1}(5.5-45 \mu \mathrm{m})$ with a FELIX bandwidth of about $1 \%$ (FWHM).

\section{COMPUTATIONAL DETAILS}

Molecular structure optimization and vibrational frequency calculations were performed with the Gaussian 09 package. ${ }^{28}$ The Tight optimization criterion (RMS force $<10^{-5}$ ) was used in all calculations. Anharmonic spectra of the molecular conformations studied were calculated with a generalized vibrational second-order perturbation theory $(\mathrm{VPT} 2)^{29}$ as implemented in Gaussian. In order to increase the accuracy of the frequency calculations, the Ultrafine grid (99 radial shells and 590 angular points per shell) was used both for numerical integration and coupled perturbed KohnSham (CPKS) computations. We have chosen the B3LYP density functional and the N07D basis set ${ }^{30}$ for the VPT2 frequency calculations, as this combination was shown to be accurate for mid-IR ${ }^{29}$ and far-IR ${ }^{31}$ vibrations of semi-rigid aromatic molecules. The potential energy distribution analysis of vibrational normal modes was performed employing the VEDA4 program. ${ }^{32}$

\section{RESULTS}

\section{A. REMPI spectra}

Experimental REMPI spectra of the molecules studied are presented in Fig. 2. The spectrum of 2-MA is dominated by a doublet band with the higher frequency peak at $35965.8 \mathrm{~cm}^{-1}$ (see inset at Fig. 2(a)). This band was assigned to the origin 0-0 transition of the trans conformer, as will be shown in Sec. IV B. The split character of the origin band and the complex low-frequency vibronic pattern in the REMPI spectra were observed earlier for similar molecular systems ${ }^{33,34}$ and originate from the presence of two populated methyl rotor states of $A$ and $E$ symmetry in the electronic ground state. The doublet band at $36054 \mathrm{~cm}^{-1}$ and two other bands outlined by blue arrows in Fig. 2(a) correspond to species different from the trans geometry of 2-MA. This was determined by our IR-UV hole-burning measurements, in which the
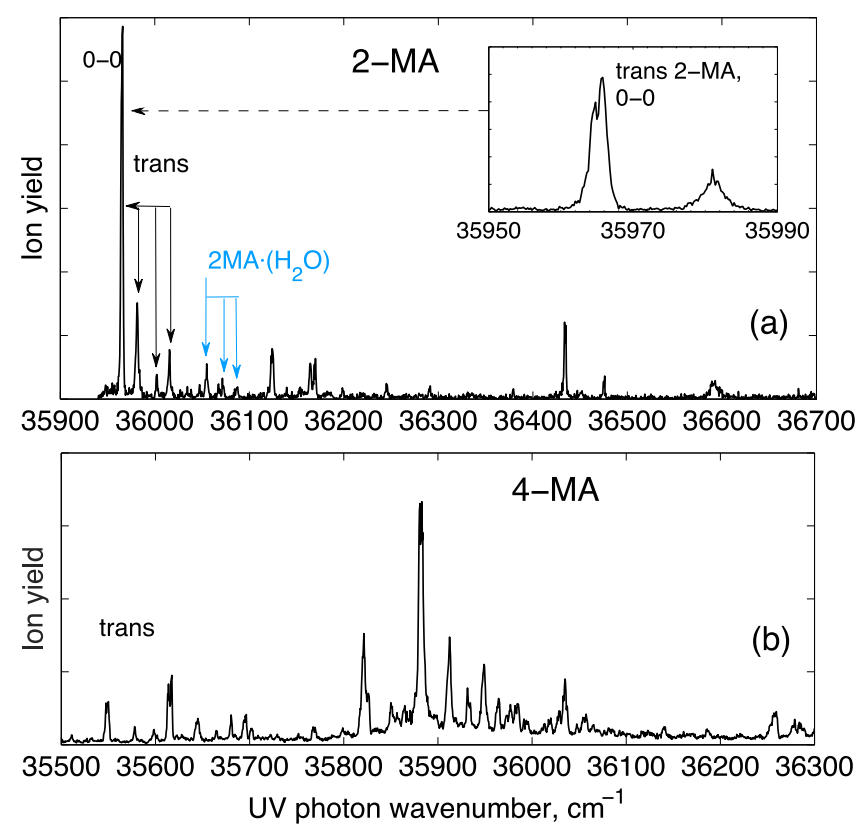

FIG. 2. (1+1)-REMPI spectra of 2-MA (a) and 4-MA (b) molecules. The origin transition of the trans conformer of 2-MA is denoted as " $0-0$." The peaks marked with blue arrows predominantly originate from UV-induced fragmentation of 2-MA $\cdot\left(\mathrm{H}_{2} \mathrm{O}\right)$ clusters.

IR wavelength was fixed to a vibrational transition of the trans conformer while the UV wavelength was scanned. The IR-UV ion-dip spectrum obtained by tuning the dye laser to these REMPI peaks was identical to that of the 2-MA. $\left(\mathrm{H}_{2} \mathrm{O}\right)$ clusters which were present in the supersonicjet molecular beam as verified by measurements of the mass spectrum. Thus we concluded these species to be 2-MA $\left(\mathrm{H}_{2} \mathrm{O}\right)$ clusters.

The spectrum of 4-MA in the range of $35500-36300 \mathrm{~cm}^{-1}$ (Fig. 2(b)) showed many vibrational progression transitions, which most likely originate from the same conformer (trans), as supported by our hole-burning measurements. The splitting due to methyl rotor states was observed for several bands, similarly to 2-MA. The intensity of the red-most peaks in the experimental spectrum of 4-MA is lower than the peaks of the blue side. This can either be an indication of a strong geometry change upon electronic excitation (putatively leading to a poor Franck-Condon overlap) or that the origin transition lies well below $35500 \mathrm{~cm}^{-1}$. We performed a time-dependent density functional theory (TDDFT) geometry optimization ${ }^{35}$ of the first electronic excited state of trans 4-MA, and, indeed, the-CO-NH- moiety altered to non-planar configuration with respect to the ring (with $\mathrm{CCNH}$ dihedral of $8.1^{\circ}$ ), and the torsional angle of para-substituent methyl group changed by $\approx 30^{\circ}$. It was not possible to determine how such a change in geometry affects the REMPI spectrum in the present experiment, but we plan to study this in detail in the future, as well as how the presence of many methyl rotors affects the REMPI spectrum and its complexity. The IR-UV ion dip spectra presented in Secs. IV B-IV $C$ were obtained by probing the origin transition of 2-MA mentioned above and the vibronic band at $35862 \mathrm{~cm}^{-1}$ of 4-MA. 


\section{B. Mid-IR spectra}

The mid-IR spectra of the conformers observed for the 2-MA and 4-MA molecules, recorded with the IRUV ion-dip technique, are presented in Fig. 3. The figure also contains theoretical spectra for the stable conformations (Fig. 1), calculated with the anharmonic VPT2 approach. The comparison with the calculated spectra strongly favors the assignment of the observed conformers of 2-MA and 4-MA to the trans geometries. The vibrations of the amide moiety are most intense in this region, with the $\mathrm{C}-\mathrm{O}$ stretch (amide $\mathrm{I}$ ) and $\mathrm{NH}$ in-plane (i.p.) bending (amide II and III) observed around 1720,1520 , and $1250 \mathrm{~cm}^{-1}$. The relatively strong vibrational bands observed in the region of $1350-1500 \mathrm{~cm}^{-1}$ are due to the deformations of the $\mathrm{CH}_{3}$ groups. As it can also be seen
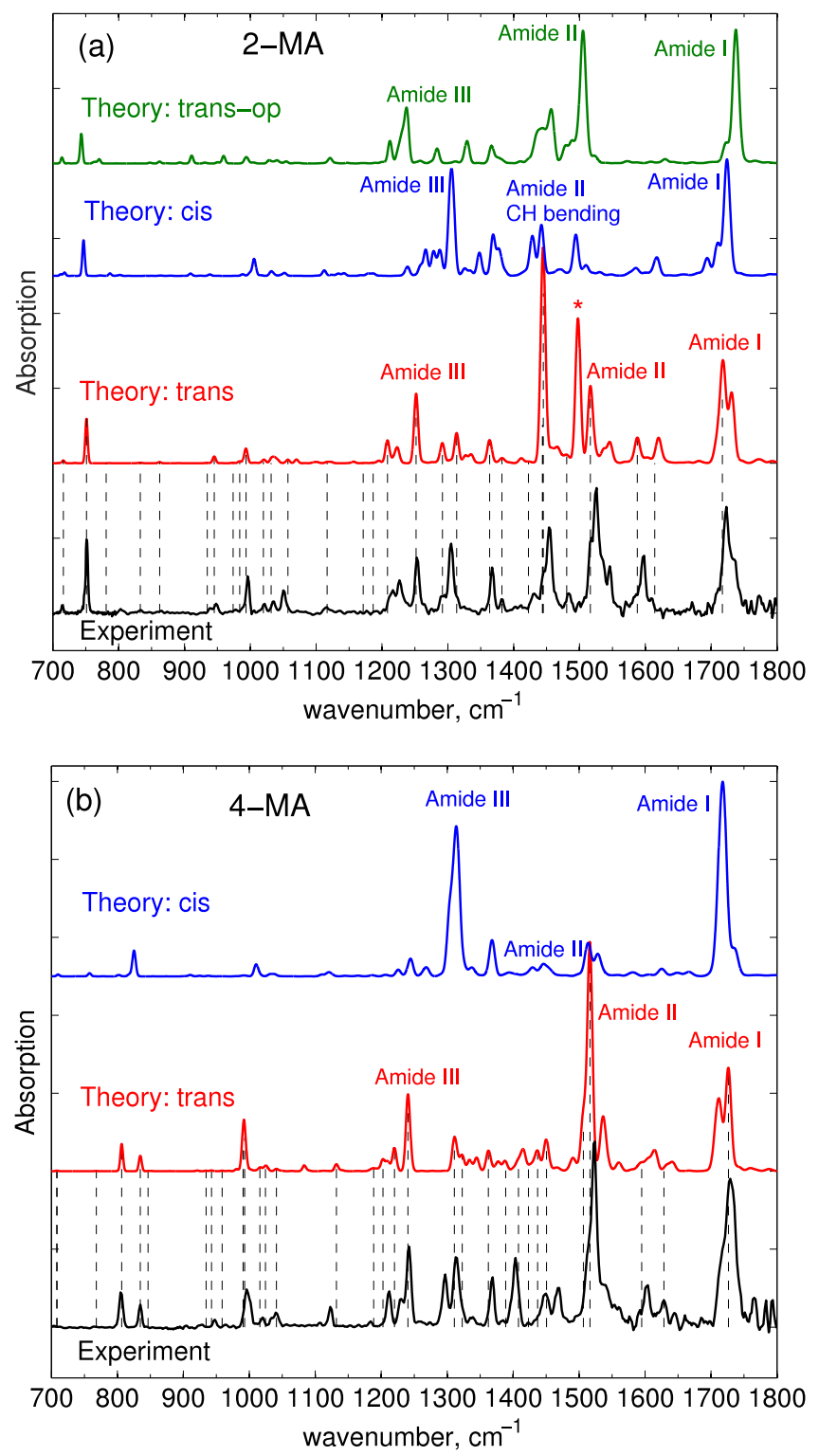

FIG. 3. Experimental mid-IR absorption spectra (in black) of the observed conformers of 2-MA (a) and 4-MA (b), which we assign to the trans geometries. The spectra calculated for all possible conformers within the VPT2 anharmonic treatment at the B3LYP/N07D level of theory are shown for comparison. The fundamental theoretical bands of the assigned trans conformers are highlighted by vertical dashed lines. from Fig. 3, the calculated VPT2 spectra (B3LYP/N07D level of theory) reproduce most of the spectral features, including weaker ones originating from combinational transitions. It is worth noting that the theoretical spectra of the cis conformers show some variations with respect to the observed trans conformer. Most prominent are the blue-shift of the amide III band in the case of cis geometry and a strong reduction of the intensity of the amide II transitions, which become much weaker than the neighboring ring and methyl $\mathrm{CH}$ bending vibrations. For the trans-op conformer of 2-MA, the calculations predict a weak red-shift of the amide II and III bands. The vibrational assignments as well as the comparison between the experimental and calculated frequencies are presented in the supplementary material of this work.

\section{Far-IR spectra}

The far-IR spectra $\left(220-740 \mathrm{~cm}^{-1}\right)$ of the observed conformers of 2-MA and 4-MA are shown in Figs. 4 and 5, as measured in our IR-UV ion-dip experiments. The figures also contain theoretical spectra for all possible conformations, calculated with the anharmonic VPT2 approach. The calculated spectra for the trans conformations are in very good agreement with the experimental spectra, thus further supporting the assignment of the observed conformers of 2-MA and 4-MA to the trans geometries made on the basis of the mid-IR data.

The strongest absorption bands in the frequency range of $220-740 \mathrm{~cm}^{-1}$ correspond to the fundamental transitions of the amide $\mathrm{V}$ vibration, as classified in the literature. ${ }^{7}$ The amide $\mathrm{V}$ vibration is associated with $\mathrm{NH}$ o.p. bending, with some contributions of the $\mathrm{CN}$ torsion motion. In the 2-MA molecule,

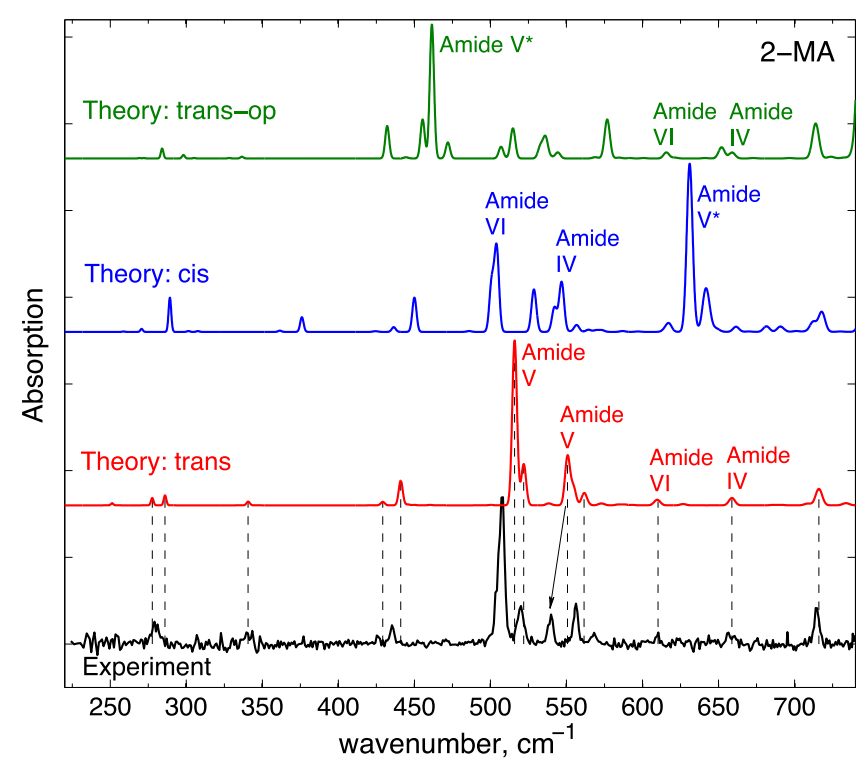

FIG. 4. Experimental far-IR absorption spectra in the range of $220-740 \mathrm{~cm}^{-1}$ (in black) for the observed conformer of 2-MA, assigned in this work to the trans geometry. The spectra of all possible conformers, calculated with the VPT2 anharmonic treatment at the B3LYP/N07D level of theory, are shown for comparison. The vertical dashed lines denote the position of the fundamental theoretical bands for the trans geometry. The marked theoretical $\mathrm{NH}$ o.p. bands (amide $\mathrm{V}^{*}$ ) were reduced in intensity by a factor of two. 


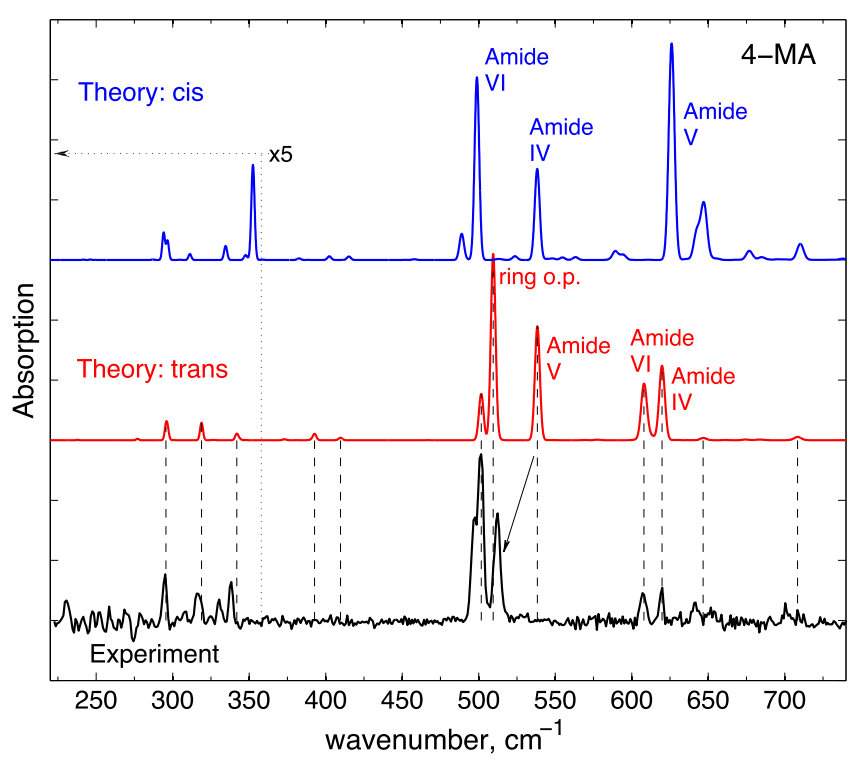

FIG. 5. Experimental far-IR absorption spectra in the range of $220-740 \mathrm{~cm}^{-1}$ (in black) for the observed conformer of 4-MA, assigned in this work to the trans geometry. The spectra of all possible conformers, calculated with the VPT2 anharmonic treatment at B3LYP/N07D level of theory, are shown for comparison. The vertical dashed lines denote the position of the fundamental theoretical bands for the trans geometry.

due to the coupling with the ring vibrations, there are two normal modes of the amide $\mathrm{V}$ character, as suggested by our frequency calculations performed with the B3LYP/N07D method. In the case of the planar trans conformation of 2-MA, we assigned the strong absorption peaks at 507.5 and $540 \mathrm{~cm}^{-1}$ to amide $\mathrm{V}$ bands with a small contribution of ring o.p. deformation. The corresponding amide $\mathrm{V}$ bands of the non-planar trans-op conformation were calculated to be red-shifted with respect to the planar trans geometry and located at 461.5 and $533 \mathrm{~cm}^{-1}$. The second band at $533 \mathrm{~cm}^{-1}$ is, however, purely due to the ring o.p. motion, while the amide $\mathrm{V}$ character is predicted to be present in the $\mathrm{CCN}$ deformation band at $432 \mathrm{~cm}^{-1}$. For trans 4-MA, the amide $\mathrm{V}$ band was observed at $512 \mathrm{~cm}^{-1}$, while the stronger band at $501 \mathrm{~cm}^{-1}$ is due to the ring o.p. deformation motion. Interestingly, for the cis geometry of the peptide link, the calculations predict a strong blue-shift $\left(>100 \mathrm{~cm}^{-1}\right)$ of the amide $\mathrm{V}$ bands with respect to the trans geometry. All far-IR vibrations associated with the peptide-CO-NH- moiety, observed and calculated for the 2-MA and 4-MA molecules, are listed in Table I.

The studied frequency range also contains transitions which correspond to amide IV and VI vibrations of the - CO-NH moiety. In the trans configuration of the peptide link, these bands are weaker than the amide $\mathrm{V}$ ones, while for the cis geometry they are predicted to be strong. Amide IV is associated with the $\mathrm{CO}$ i.p. bending and $\mathrm{CC}$ stretch motions, with a small contribution from $\mathrm{CNC}$ deformation (see Fig. 1(b)). Amide VI involves CO o.p. bending, with some o.p. displacement of the NH group. In the experimental spectra of trans 2-MA, the amide IV and VI bands appeared at 656 and $610 \mathrm{~cm}^{-1}$, respectively, which is in good agreement with the theoretical data. The calculated frequencies of the same bands in the case of the non-planar trans-op conformer only slightly deviate from the trans one. In the 4-MA isomer, the amide IV and VI bands for the trans geometry appeared at 620 and $607 \mathrm{~cm}^{-1}$, respectively, hence indicating the red-shift with respect to the 2-MA isomer. The shift is most likely due to the different reduced masses of the corresponding normal modes in different isomers. The amide IV and VI bands of both 2-MA and 4-MA in the cis configuration are calculated to be strongly red-shifted and enhanced in intensity compared with the trans data.

Other characteristic vibrations of the peptide $-\mathrm{CO}-\mathrm{NH}-$ moiety in the far-IR are associated with CCO i.p. bending, and CCN (or CNC) i.p. bending (see Table I). These vibrations have non-local nature, and for the 2-MA and 4-MA in the trans geometry they show variations in frequency, mostly due to the contributions from other types of the phenyl ring and methyl group motions. Among the $\mathrm{CCN}$ and $\mathrm{CNC}$ i.p. bending vibrations of the trans and trans-op geometries of 2-MA, only

TABLE I. Experimental and theoretical frequencies of vibrations which are characteristic for the peptide -CO-NH- moiety in 2-MA and 4-MA. The theoretical values are from the VPT2 frequency analysis at the B3LYP/N07D level of theory. The calculated harmonic frequencies and available experimental ${ }^{36}$ data for N-Methylacetamide (NMA) and its dimer are shown for comparison.

\begin{tabular}{|c|c|c|c|c|c|c|c|c|c|c|c|c|c|c|}
\hline & \multicolumn{2}{|c|}{ trans 2-MA } & \multicolumn{2}{|c|}{ trans-op 2-MA } & \multicolumn{2}{|c|}{ cis 2-MA } & \multicolumn{2}{|c|}{ trans 4-MA } & \multicolumn{2}{|c|}{ cis 4-MA } & \multicolumn{2}{|c|}{ NMA } & \multicolumn{2}{|c|}{ NMA dimer } \\
\hline & Exp. & Theory & Exp. & Theory & Exp. & Theory & Exp. & Theory & Exp. & Theory & Exp. & Theory & Exp. & Theory \\
\hline Amide IV & 656 & 658.8 & $\ldots$ & 659.0 & $\ldots$ & 546.8 & 620 & 619.8 & $\ldots$ & 538.2 & $\begin{array}{c}619^{\mathrm{a}} \\
\ldots\end{array}$ & $\begin{array}{l}623.0 \\
627.9\end{array}$ & $\ldots$ & 624.9 \\
\hline Amide VI & 610 & 610.2 & $\ldots$ & 615.7 & $\ldots$ & 504.1 & 607 & 608.0 & $\ldots$ & 498.8 & $\begin{array}{c}658^{\mathrm{a}} \\
\ldots\end{array}$ & $\begin{array}{l}630.8 \\
630.0\end{array}$ & $\ldots$ & 597.6 \\
\hline Amide V & $\begin{array}{l}507.5 \\
540\end{array}$ & $\begin{array}{l}515.9 \\
550.7\end{array}$ & $\begin{array}{l}\cdots \\
\ldots\end{array}$ & $\begin{array}{c}461.5 \\
\ldots\end{array}$ & $\begin{array}{l}\ldots \\
\ldots\end{array}$ & $\begin{array}{l}631.0 \\
641.0\end{array}$ & $\begin{array}{r}512 \\
\ldots\end{array}$ & $\begin{array}{c}538.3 \\
\ldots\end{array}$ & $\begin{array}{l}\ldots \\
\ldots\end{array}$ & $\begin{array}{l}626.1 \\
645.5\end{array}$ & $\begin{array}{c}439^{\mathrm{a}} \\
\ldots\end{array}$ & $\begin{array}{c}458.6 \\
\ldots\end{array}$ & $\begin{array}{l}\ldots \\
\ldots\end{array}$ & $\begin{array}{l}716.9 \\
423.8\end{array}$ \\
\hline CCO i.p. bend & 556 & 561.6 & $\ldots$ & 576.8 & $\ldots$ & 556.7 & $\ldots$ & $\ldots$ & $\ldots$ & 589.2 & $\ldots$ & $\ldots$ & $\ldots$ & \\
\hline CCN def. & $\begin{array}{l}520 \\
426 \\
340\end{array}$ & $\begin{array}{l}521.9 \\
429.2 \\
340.6\end{array}$ & $\begin{array}{l}\cdots \\
\cdots \\
\cdots\end{array}$ & $\begin{array}{l}514.8 \\
432.1^{\mathrm{b}} \\
336.6\end{array}$ & $\begin{array}{l}\cdots \\
\cdots \\
\ldots\end{array}$ & $\begin{array}{l}500.8 \\
436.4 \\
376.0\end{array}$ & $\begin{array}{r}497 \\
\ldots \\
316\end{array}$ & $\begin{array}{l}501.6 \\
392.7 \\
318.9\end{array}$ & $\begin{array}{l}\cdots \\
\cdots \\
\ldots\end{array}$ & $\begin{array}{l}488.9 \\
402.4 \\
352.2\end{array}$ & $\begin{array}{c}\ldots \\
429^{\mathrm{a}} \\
279^{\mathrm{a}}\end{array}$ & $\begin{array}{c}\ldots \\
433.7 \\
287.0\end{array}$ & $\begin{array}{l}\cdots \\
\cdots \\
\ldots\end{array}$ & \\
\hline $\mathrm{CNC}$ def. $+\mathrm{CH}_{3}$ i.p. bending & 279 & 277.6 & $\cdots$ & 298.1 & $\ldots$ & 270.6 & 294.5 & 295.6 & $\ldots$ & 296.6 & $\ldots$ & $\ldots$ & $\ldots$ & \\
\hline $\mathrm{CNC}$ def. $+\mathrm{CH}_{3}$ o.p. bending & 282 & 286.0 & $\ldots$ & 284.1 & $\ldots$ & 289.2 & 338 & 341.9 & $\ldots$ & 334.6 & $\ldots$ & $\ldots$ & $\ldots$ & \\
\hline
\end{tabular}

${ }^{\mathrm{a}}$ From Ref. 36.

${ }^{\mathrm{b}}$ This CCN deformation vibration of trans-op 2-MA also has a small amide V contribution. 
CNC deformation with the methyl i.p. bending are expected to have significant variations in frequency (see Table I). However, according to the calculations for the cis geometry, the CCN deformation vibrations show some variations with respect to the trans geometry of 2-MA and 4-MA.

\section{DISCUSSION}

To begin with, we shall discuss the predominant abundance of the trans conformations of the 2-MA and 4-MA molecules, and absence of other conformations in our supersonic-jet experiments. The literature data for similar molecular systems include several gas-phase studies on the conformational preferences of the peptide - $\mathrm{CO}-\mathrm{NH}-$ moiety in $\mathrm{N}$-phenylamides. ${ }^{33,37-41}$ Among them, the formanilide molecule with a hydrogen attached to the $\mathrm{CO}$ group was observed in both the trans and cis configurations, ${ }^{33,37,38}$ with the trans conformation being more abundant than cis $(93.5 \%$ and $6.5 \%$ abundance, respectively). The replacement of the amidic hydrogen with a methyl group in $N$-methylformanilide led to an increased abundance of the cis geometry, but also the trans amide group was twisted out of plane with respect to the phenyl ring. ${ }^{33}$ For the acetanilide molecule, with a methyl group attached to the $\mathrm{CO}$ group, only the trans configuration was detected by vibrationally resolved electronic spectroscopy. ${ }^{33,37,39}$ However, in a subsequent study, the cis configuration was found with the help of microwave spectroscopy, ${ }^{40}$ despite the fact that the $c i s$ species were twenty times less abundant than the trans species.

The 2-MA and 4-MA molecules studied have similar chemical composition to acetanilide, with a difference that the hydrogen atom of the aromatic ring is substituted by a methyl group in the ortho- and para-position, respectively. Similar to acetanilide, the gas-phase 2-MA and 4-MA molecules adopt preferentially the trans conformation as indicated by our IR-UV ion-dip and hole-burning measurements, as well as verified by the theoretical calculations (Figs. 3-5). The hole-burning measurements performed for 2-MA and 4-MA identified only the trans conformers. Moreover, the search for 2-MA REMPI transitions in the range of $35200-36700 \mathrm{~cm}^{-1}$ did not show any peaks originating from other conformers than trans.

According to our DFT calculations at the B3LYP/N07D level, for the trans geometry of 4-MA, the amide group is planar with respect to the ring, and the hydrogen of the para-methyl group has a CCCH dihedral angle of $90^{\circ}$ (Fig. 1). The cis geometry is also stable, but it is $10.6 \mathrm{~kJ} / \mathrm{mol}$ higher in energy than the trans geometry, which can result in a very low abundance in our gas-phase measurements. In the case of 2-MA, three different stable geometries are possible: the planar trans is most stable, the non-planar trans-op is higher in energy by $6 \mathrm{~kJ} / \mathrm{mol}$, and cis is even higher by $8.15 \mathrm{~kJ} / \mathrm{mol}$ (B3LYP/N07D level). Similar to the case of 4-MA, the trans-op and cis conformers of 2-MA can have a low abundance, despite that their energies above the planar trans structure are lower than in 4-MA. The amide moiety of the trans-op conformer is twisted out of plane with respect to the phenyl ring by $\approx 60^{\circ}$, due to steric interactions between the adjacent carbonyl oxygen and the methyl group in the ortho-position. The amide moiety in the cis configuration of the peptide link is also twisted out of plane by $\approx 48^{\circ}$ for both 2-MA and 4-MA. The reason for this is a steric interaction between the methyl group and the hydrogen atom of the phenyl ring.

The measured far-IR spectra of the observed trans conformations of 2-MA and 4-MA are in very good agreement with the DFT frequency calculations based on the anharmonic VPT2 approach. This further confirms our theoretical data obtained for the other conformations, which we can use for a comparative analysis, at least qualitatively, between the vibrational signatures of the different conformations. For example, the amide $\mathrm{V}$ band in the trans-op geometry of 2-MA is predicted to be red-shifted by $\approx 50 \mathrm{~cm}^{-1}$ versus the trans geometry, which correlates with the structural differences between the two conformations. First, due to the resonance interactions between the ring and - $\mathrm{CO}-\mathrm{NH}-$ moiety in the case of the planar trans conformation, the force constants of $\mathrm{NH}$ o.p. bending motion are increased because this vibration distorts the resonantly stabilized planar geometry. The trans-op conformation, however, has a twisted geometry $\left(\approx 60^{\circ}\right)$ of the acetamide group with respect to the aromatic ring, and hence the force constants are lower. Second, the - $\mathrm{CO}-\mathrm{NH}-$ moiety in the trans-op conformation deviates from planarity by $\approx 10^{\circ}$ (B3LYP/N07D geometry) thus further reducing the force constants. Such deviations from planarity may also take place in polypeptide structures, ${ }^{25,26}$ and the amide $\mathrm{V}$ vibrations might be very diagnostic in order to study them. Moreover, in the case of weak $\mathrm{NH} \cdots \pi$ interactions between the aromatic groups of amino acids and nearby amide hydrogen, ${ }^{42}$ the amide $\mathrm{V}$ vibration can be diagnostic as well.

The frequency calculations show that the amide $\mathrm{V}$ bands of the cis isomers are strongly blue-shifted with respect to the trans isomer, by 123.5 and $88 \mathrm{~cm}^{-1}$ for 2-MA and 4-MA, respectively, (see Figs. 4-5 and Table I). Moreover, the amide IV and VI bands of the cis form are predicted to be strongly red-shifted compared with the trans form. Such changes in the fundamental amide frequencies most likely originate from the different geometry of the peptide link and reduced masses of the corresponding normal modes. It is worth noting that the amide $\mathrm{V}$ frequency of the cis peptide link itself is higher by $54 \mathrm{~cm}^{-1}$ than for the trans form, as calculated for the model molecule NMA at B3LYP/N07D level. Moreover, the optimized geometries of the cis 2-MA and 4-MA show that the aromatic ring induces the geometric constraints which reduce the distance between the $\mathrm{O}$ and $\mathrm{H}$ atoms of the peptide moiety to 2.365 and $2.369 \AA$ correspondingly, in comparison with the cis-NMA value of $2.415 \AA$. This structural change can be a reason for the shifts of the amide IV-VI bands. The large differences between the amide IV-VI bands of trans and $c i$ isomers indicate that far-IR spectroscopy can be used to analyze the fraction of cis-type peptide links. Despite the fact that the majority of the peptide links in proteins and peptides are of trans-type, this finding can be important for the structural characterization of cyclic peptides, where links of the cis-type can be present. ${ }^{43-48}$ In the current study, it was not possible to measure the far-IR spectrum of the 2-MA cis conformer because of its low abundance under the supersonic jet conditions. A potential candidate for future studies of 
the sensitivity of the far-IR amide bands is a formanilide molecule, which was observed both in trans and cis form. ${ }^{37}$ The dipeptide Phe-Ser in linear and cyclic form ${ }^{47}$ is another candidate for such investigations.

The most abundant secondary structures in peptides and proteins such as $\alpha$-helices and $\beta$-sheets are stabilized by hydrogen bonding $(\mathrm{NH} \cdots \mathrm{O}-\mathrm{C})$. Since most of the amide bands are altered by the presence of hydrogen bonding, IR spectroscopy of amide vibrations can be used to study secondary protein structures. For example, the amide I bands manifest a red-shift in frequency, while amide II-III show a blue-shift. To study the effect of hydrogen bonding on the amide IV-VI bands, we performed frequency calculations on a simple peptide model molecule, NMA, and its hydrogen bonded dimers and trimers. The theoretical results for NMAdimer show that upon $\mathrm{NH} \cdots \mathrm{O}-\mathrm{C}$ hydrogen bonding, the amide $\mathrm{V}$ frequency shifts to the blue by $258 \mathrm{~cm}^{-1}$, from 459 to $717 \mathrm{~cm}^{-1}$ at the B3LYP/N07D level of theory, which amounts to more than $50 \%$ of the frequency of the NMA-monomer without hydrogen bonding. The corresponding blue-shift for the NMA-trimer with two hydrogen bonds is even larger, $294 \mathrm{~cm}^{-1}$. These results are also supported by available experimental data on matrix and condensed-phase spectra for NMA. ${ }^{7,36,49,50}$ Such a large alternation in the frequency of the amide $\mathrm{V}$ band makes it a very promising candidate for an accurate determination of both secondary structures of polypeptides and the strength of the hydrogen bonds involved.

\section{CONCLUSIONS}

In this work, an experimental study of low-frequency vibrations of isolated 2- and 4-Methylacetanilide molecules was combined with anharmonic VPT2 calculations. The planar geometries with the trans configuration of the peptide link were found to be strongly favored under the supersonic-jet expansion conditions. The experimental and theoretical data show that the amide IV-VI vibrations of the - $\mathrm{CO}-\mathrm{NH}-$ moiety can be very sensitive to the structure of the peptide link and the molecular backbone, further supporting the previously demonstrated conformer-specificity of such vibrations. ${ }^{23}$ For the cis-configuration of the-CO-NH-moiety, our calculations predict a strong blue-shift of the amide $\mathrm{V}$ bands with respect to the trans geometry and a strong red-shift of the amide IV and VI bands. This feature can enable a far-IR characterization of cyclic peptides where the cis-peptide links can exist. Furthermore, the amide $\mathrm{V}$ vibration manifests strong blue-shifts upon hydrogen bond formation on the NH moiety, thus being a sensitive characteristic of the hydrogen bond strength and secondary structures of polypeptides stabilized by hydrogen bonding.

\section{SUPPLEMENTARY MATERIAL}

The supplementary material contains experimental and theoretical vibrational frequencies of the trans 2-MA and 4-MA conformers observed experimentally, as well as theoretical frequencies for the trans-op conformer of 2-MA. The theoretical analysis is performed in the harmonic approximation and with the VPT2 approach using the B3LYP/N07D level of theory.

\section{ACKNOWLEDGMENTS}

We acknowledge the Swedish Research Council (VR) (Grant No. 621-2011-4043), the Knut and Alice Wallenberg Foundation (Sweden), and the Dutch Foundation for Fundamental Research on Matter (FOM) for financial support. The research leading to these results has received funding from the European Community's Seventh Framework Programme (No. FP7/2007-2013) under Grant Agreement No. 312284. The authors highly appreciate the skillful assistance of the staff at the FELIX laboratory. The calculations were sponsored by NWO Physical Sciences (EW) by granting access to the supercomputer facilities at SurfSara (Project No. MP-264-13).

${ }^{1}$ Gas-Phase IR Spectroscopy and Structure of Biological Molecules, edited by A. M. Rijs and J. Oomens, Topics in Current Chemistry Vol. 364 (Springer International Publishing, Switzerland, 2015), pp. 1-406 and references therein.

${ }^{2}$ D. J. Bakker, A. Peters, V. Yatsyna, V. Zhaunerchyk, and A. M. Rijs, J. Phys. Chem. Lett. 7, 1238 (2016).

${ }^{3}$ R. J. Falconer and A. G. Markelz, J. Infrared, Millimeter, Terahertz Waves 33, 973 (2012).

${ }^{4}$ T. Ding, A. P. Middelberg, T. Huber, and R. J. Falconer, Vib. Spectrosc. 61, 144 (2012).

${ }^{5}$ S. Perticaroli, D. Russo, M. Paolantoni, M. A. Gonzalez, P. Sassi, J. D. Nickels, G. Ehlers, L. Comez, E. Pellegrini, D. Fioretto, and A. Morresi, Phys. Chem. Chem. Phys. 17, 11423 (2015).

${ }^{6}$ B. Born, S. J. Kim, S. Ebbinghaus, M. Gruebele, and M. Havenith, Faraday Discuss. 141, 161 (2009).

${ }^{7}$ J. Bandekar, Biochimica et Biophysica Acta (BBA) - Protein Structure and Molecular Enzymology 1120, 123 (1992).

${ }^{8}$ T. Miyazava, K. Fukusima, S. Sugano, and Y. Masuda, in Conformation of Biopolymers, edited by G. Ramachandran (Academic Press, 1967), pp. 557-568.

${ }^{9}$ Y. Masuda, K. Fukushima, T. Fujii, and T. Miyazawa, Biopolymers 8, 91 (1969).

${ }^{10}$ K. Itoh, T. Nakahara, T. Shimanouchi, M. Oya, K. Uno, and Y. Iwakura, Biopolymers 6, 1759 (1968).

${ }^{11}$ K. Itoh, T. Shimanouchi, and M. Oya, Biopolymers 7, 649 (1969).

${ }^{12}$ K. Itoh, M. Oya, and T. Shimanouchi, Biopolymers 11, 1137 (1972).

${ }^{13}$ K. Itoh and H. Katabuchi, Biopolymers 11, 1593 (1972).

${ }^{14} \mathrm{~K}$. Itoh and H. Katabuchi, Biopolymers 12, 921 (1973).

${ }^{15}$ J. Bandekar and G. Zundel, Spectrochim. Acta, Part A 38, 815 (1982).

${ }^{16}$ H. Kellouai, M. Barthes, G. Page, J. Moret, and J. L. Sauvajol, J. Biol. Phys. 21, 25 (1995).

${ }^{17}$ P. Papanek, J. E. Fischer, and N. S. Murthy, Macromolecules 35, 4175 (2002).

${ }^{18}$ T. Ding, T. Huber, A. P. Middelberg, and R. J. Falconer, J. Phys. Chem. A 115, 11559 (2011).

${ }^{19}$ P. Carcabal, R. T. Kroemer, L. C. Snoek, J. P. Simons, J. M. Bakker, I. Compagnon, G. Meijer, and G. von Helden, Phys. Chem. Chem. Phys. 6, 4546 (2004).

${ }^{20}$ J. M. Bakker, L. M. Aleese, G. Meijer, and G. von Helden, Phys. Rev. Lett. 91, 203003 (2003)

${ }^{21}$ M. Cirtog, A. M. Rijs, Y. Loquais, V. Brenner, B. Tardivel, E. Gloaguen, and M. Mons, J. Phys. Chem. Lett. 3, 3307 (2012).

${ }^{22}$ S. Jaeqx, J. Oomens, A. Cimas, M.-P. Gaigeot, and A. M. Rijs, Angew. Chem., Int. Ed. 53, 3663 (2014).

${ }^{23}$ J. Mahe, S. Jaeqx, A. M. Rijs, and M.-P. Gaigeot, Phys. Chem. Chem. Phys. 17, 25905 (2015).

${ }^{24}$ M.-P. Gaigeot, Phys. Chem. Chem. Phys. 12, 3336 (2010).

${ }^{25}$ D. S. Berkholz, C. M. Driggers, M. V. Shapovalov, R. L. Dunbrack, and P. A. Karplus, Proc. Natl. Acad. Sci. U. S. A. 109, 449 (2012).

${ }^{26}$ R. Improta, L. Vitagliano, and L. Esposito, PLoS ONE 6, 1 (2011).

${ }^{27}$ A. M. Rijs and J. Oomens, IR Spectroscopic Techniques to Study Isolated Biomolecules, Topics in Current Chemistry Vol. 364 (Springer International Publishing, Switzerland, 2015), pp. 1-42. 
${ }^{28}$ M. J. Frisch, G. W. Trucks, H. B. Schlegel, G. E. Scuseria, M. A. Robb, J. R. Cheeseman, G. Scalmani, V. Barone, B. Mennucci, G. A. Petersson, H. Nakatsuji, M. Caricato, X. Li, H. P. Hratchian, A. F. Izmaylov, J. Bloino, G. Zheng, J. L. Sonnenberg, M. Hada, M. Ehara, K. Toyota, R. Fukuda, J. Hasegawa, M. Ishida, T. Nakajima, Y. Honda, O. Kitao, H. Nakai, T. Vreven, J. A. Montgomery, Jr., J. E. Peralta, F. Ogliaro, M. Bearpark, J. J. Heyd, E. Brothers, K. N. Kudin, V. N. Staroverov, R. Kobayashi, J. Normand, K. Raghavachari, A. Rendell, J. C. Burant, S. S. Iyengar, J. Tomasi, M. Cossi, N. Rega, J. M. Millam, M. Klene, J. E. Knox, J. B. Cross, V. Bakken, C. Adamo, J. Jaramillo, R. Gomperts, R. E. Stratmann, O. Yazyev, A. J. Austin, R. Cammi, C. Pomelli, J. W. Ochterski, R. L. Martin, K. Morokuma, V. G. Zakrzewski, G. A. Voth, P. Salvador, J. J. Dannenberg, S. Dapprich, A. D. Daniels, Ö. Farkas, J. B. Foresman, J. V. Ortiz, J. Cioslowski, and D. J. Fox, GaUsSIAN 09, Revision D.01, Gaussian, Inc., Wallingford, CT, 2009.

${ }^{29}$ V. Barone, M. Biczysko, and J. Bloino, Phys. Chem. Chem. Phys. 16, 1759 (2014), and references therein.

${ }^{30}$ V. Barone, P. Cimino, and E. Stendardo, J. Chem. Theory Comput. 4, 751 (2008).

${ }^{31}$ V. Yatsyna, D. J. Bakker, R. Feifel, A. M. Rijs, and V. Zhaunerchyk, Phys. Chem. Chem. Phys. 18, 6275 (2016).

${ }^{32}$ M. H. Jamroz, Spectrochim. Acta, Part A 114, 220 (2013).

${ }^{33}$ V. P. Manea, K. J. Wilson, and J. R. Cable, J. Am. Chem. Soc. 119, 2033 (1997).

${ }^{34}$ W. Y. Sohn, S.-i. Ishiuchi, M. Miyazaki, J. Kang, S. Lee, A. Min, M. Y. Choi, H. Kang, and M. Fujii, Phys. Chem. Chem. Phys. 15, 957 (2013).

${ }^{35}$ F. Furche and R. Ahlrichs, J. Chem. Phys. 117, 7433 (2002).
${ }^{36}$ S. Ataka, H. Takeuchi, and M. Tasumi, J. Mol. Struct. 113, 147 (1984).

${ }^{37}$ M. Miyazaki, J. Saikawa, H. Ishizuki, T. Taira, and M. Fujii, Phys. Chem. Chem. Phys. 11, 6098 (2009).

${ }^{38}$ J. A. Dickinson, M. R. Hockridge, E. G. Robertso, and J. P. Simons, J. Phys. Chem. A 103, 6938 (1999).

${ }^{39}$ S. Ullrich and K. Müller-Dethlefs, J. Phys. Chem. A 106, 9181 (2002).

${ }^{40}$ C. Cabezas, M. Varela, W. Caminati, S. Mata, J. Lopez, and J. Alonso, J. Mol. Spectrosc. 268, 42 (2011).

${ }^{41}$ M. Varela, C. Cabezas, J. C. Lopez, and J. L. Alonso, J. Phys. Chem. A 117, 13275 (2013).

${ }^{42}$ G. Toth, C. R. Watts, R. F. Murphy, and S. Lovas, Proteins: Struct., Funct., Bioinf. 43, 373 (2001).

${ }^{43}$ D. F. Mierke, T. Yamazaki, O. E. Said-Nejad, E. R. Felder, and M. Goodman, J. Am. Chem. Soc. 111, 6847 (1989).

${ }^{44}$ M. T. Oakley and R. L. Johnston, J. Chem. Theory Comput. 9, 650 (2013).

${ }^{45}$ S. Wiedemann, A. Metsala, D. Nolting, and R. Weinkauf, Phys. Chem. Chem. Phys. 6, 2641 (2004).

${ }^{46}$ A. P. Wickrama Arachchilage, F. Wang, V. Feyer, O. Plekan, and K. C. Prince, J. Chem. Phys. 136, 124301 (2012).

${ }^{47}$ A. G. Abo-Riziq, B. Crews, J. E. Bushnell, M. P. Callahan, and M. S. D. Vries, Mol. Phys. 103, 1491 (2005).

${ }^{48}$ K. Schwing, C. Reyheller, A. Schaly, S. Kubik, and M. Gerhards, Chem. Phys. Chem. 12, 1981 (2011).

${ }^{49}$ F. Fillaux and M. Baron, Chem. Phys. 62, 275 (1981).

${ }^{50}$ W. A. Herrebout, K. Clou, and H. O. Desseyn, J. Phys. Chem. A 105, 4865 (2001). 